\title{
OPINION
}

\section{Pathogenesis and diagnostic criteria for rickets and osteomalacia - Proposal by an expert panel supported by Ministry of Health, Labour and Welfare, Japan, The Japanese Society for Bone and Mineral Research and The Japan Endocrine Society}

\author{
Seiji Fukumoto ${ }^{1)}$, Keiichi Ozono ${ }^{2)}$, Toshimi Michigami ${ }^{3)}$, Masanori Minagawa ${ }^{4)}$, Ryo Okazaki ${ }^{5)}$, \\ Toshitsugu Sugimoto ${ }^{6}$, Yasuhiro Takeuchi ${ }^{7)}$ and Toshio Matsumoto ${ }^{1)}$ \\ 1) Fujii Memorial Institute of Medical Sciences, Tokushima University, Tokushima 770-8503, Japan \\ ${ }^{2)}$ Department of Pediatrics, Osaka University Graduate School of Medicine, Suita 565-0871, Japan \\ ${ }^{3)}$ Department of Bone and Mineral Research, Research Institute, Osaka Medical Center for Maternal and Child Health, Izumi \\ 594-1101, Japan \\ 4) Department of Endocrinology, Chiba Children's Hospital, Chiba 266-0007, Japan \\ ${ }^{5)}$ Third Department of Medicine, Teikyo University Chiba Medical Center, Ichihara 299-0111, Japan \\ 6) Internal Medicine 1, Shimane University Faculty of Medicine, Izumo 693-8501, Japan \\ ${ }^{7)}$ Division of Endocrinology, Toranomon Hospital Endocrine Center, Tokyo 105-8470, Japan
}

\begin{abstract}
Rickets and osteomalacia are diseases characterized by impaired mineralization of bone matrix. Recent investigations revealed that the causes for rickets and osteomalacia are quite variable. While these diseases can severely impair the quality of life of the affected patients, rickets and osteomalacia can be completely cured or at least respond to treatment when properly diagnosed and treated according to the specific causes. On the other hand, there are no standard criteria to diagnose rickets or osteomalacia nationally and internationally. Therefore, we summarize the definition and pathogenesis of rickets and osteomalacia, and propose the diagnostic criteria and a flowchart for the differential diagnosis of various causes for these diseases. We hope that these criteria and flowchart are clinically useful for the proper diagnosis and management of patients with these diseases.
\end{abstract}

Key words: Vitamin D, Hypophosphatemia, Hyopcalcemia, FGF23

RICKETS and osteomalacia are diseases characterized by impaired mineralization of bone matrix $[1,2]$. While rickets and osteomalacia are caused by the same etiologies, rickets develops before the closure of the growth plates. These diseases have been classified as metabolic bone diseases and endocrinologists have not considered these as endocrine diseases. Historically, nutritional vitamin D deficient rickets and osteomalacia were clinically very important [3]. Studies about this vitamin $\mathrm{D}$ deficient diseases led to the identification of 25-hydroxyvitain D [25(OH)D] and 1,25-dihydroxyvitamin $\mathrm{D}\left[1,25(\mathrm{OH})_{2} \mathrm{D}\right]$. Since then, many causes of rickets and osteomalacia have been identified. Especially,

Submitted May 19, 2015; Accepted Jun. 9, 2015 as EJ15-0289 Released online in J-STAGE as advance publication Jul. 4, 2015

Correspondence to: Seiji Fukumoto, Fujii Memorial Institute of Medical Sciences, Tokushima University, 3-18-15 Kuramoto-cho, Tokushima 770-8503, Japan. E-mail: fukumoto-tky@umin.ac.jp recent studies established that fibroblast growth factor 23 (FGF23) is a phosphotropic hormone produced by bone and excessive actions of FGF23 cause several kinds of hypophosphatemic rickets and osteomalacia [4]. These results indicate that at least some kinds of hypophosphatemic rickets and osteomalacia are now considered to be endocrine diseases. Furthermore, it has been proposed that FGF23 measurement is useful for the differential diagnosis of hypophosphatemic diseases [5]. Therefore, it may be possible to newly classify causes of rickets and osteomalacia based on the pathophysiology utilizing these new findings.

There are no standard criteria to diagnose rickets or osteomalacia nationally and internationally. Since rickets and osteomalacia are not common lifestyle diseases, it may be difficult for general health professionals to properly diagnose and manage patients with these diseases. As impaired mineralization results in 
low bone mineral density measured by dual energy $\mathrm{X}$-ray absorptiometry, osteomalacia must be discriminated from osteoporosis, a much commoner disease. Bone deformities observed in patients with rickets may lead to a wrong diagnosis like skeletal dysplasia. On the other hand, rickets and osteomalacia can severely impair the quality of life of the affected patients without proper diagnosis and management. From these backgrounds, it is clinically important to establish diagnostic criteria for rickets and osteomalacia that can be easily used by health professionals. In this review, we briefly summarize the definition and pathogenesis of rickets and osteomalacia, and propose the diagnostic criteria and investigation methods for the differential diagnosis of various causes for these diseases.

\section{Definition and Clinical Presentations}

Bone is a hard tissue created by deposition of hydroxyapatite crystals $\left[\mathrm{Ca}_{10}\left(\mathrm{PO}_{4}\right)_{6}(\mathrm{OH})_{2}\right]$ on matrix proteins produced by osteoblasts. Because of the impairment of this mineralization of matrix proteins, unmineralized bone matrix (osteoid) increases in rickets and osteomalacia $[1,2]$. Of these, rickets develops in children and osteomalacia is a disease in adulthood. Patients with rickets present several symptoms and signs including growth retardation, bone deformities such as genu valgum and varum, spinal curvature, craniotabes, open fontanels, rachitic rosary and joint swelling $[1,2]$. Patients with osteomalacia may complain of bone pain and have muscle weakness, pigeon chest, spinal curvature and pseudofractures (Looser's zone) [1, 2].

\section{Pathogenesis and Causes}

Several drugs such as aluminum and etidronate directly inhibit mineralization of bone matrix proteins. In most other cases with rickets or osteomalacia, chronic hypophosphatemia, hypocalcemia, or both are present (Table 1). Hydroxyapatite crystals are formed in matrix vesicles produced by osteoblasts from calcium ion and phosphate. Hypophosphatemia or hypocalcemia is believed to impair mineralization by decreasing calcium $\mathrm{x}$ phosphate product.

Serum phosphate level is maintained by intestinal phosphate absorption, renal phosphate handling and phosphate movement between extracellular fluid and bone or intracellular fluid. Of these, chronic hypophosphatemia resulting in rickets and osteomalacia
Table 1 Causes of rickets/osteomalacia

\begin{tabular}{l}
\hline Hypophosphatemia \\
Impaired actions of vitamin D metabolites \\
Vitamin D deficiency \\
Drugs (Diphenylhydantoin, Rifampicin, etc.) \\
$\quad$ Vitamin D-dependent rickets type $1^{1)}$ \\
$\quad$ Vitamin D-dependent rickets type $2^{2)}$ \\
Renal tubular dysfunction \\
$\quad$ Hereditary hypophosphatemic rickets with hypercalciuria ${ }^{3)}$ \\
Fanconi syndrome \\
$\quad$ Dent disease \\
$\quad$ Renal tubular acidosis \\
$\quad$ Drugs (Ifosfamide, Adefovir pivoxil, Valproic acid, etc.) \\
FGF23-related hypophosphatemic rickets/osteomalacia (Table 2) \\
Phosphate depletion \\
Phosphate deficiency \\
$\quad$ Malabsorption... \\
Hypocalcemia \\
Some cases of vitamin D deficiency \\
Vitamin D-dependent rickets type $1^{1)}$ \\
Vitamin D-dependent rickets type $2^{2)}$ \\
Impaired mineralization from other causes \\
Drugs (Aluminum, Etidronate, etc.) \\
1) Mutations in $C Y P 27 B 1$ gene, autosomal recessive \\
Mutations in $V D R$ gene, autosomal recessive \\
Mutions in $C L C N 5$ gene, X-linked recessive
\end{tabular}

is usually caused by impaired intestinal phosphate absorption and/or renal phosphate wasting. Phosphate is abundant in food and phosphate deficiency does not occur in healthy subjects taking usual food. Phosphate deficiency may be observed in some patients suffering from malnutrition, malabsorption syndrome and so on.

$1,25(\mathrm{OH})_{2} \mathrm{D}$ enhances intestinal phosphate and calcium absorption, and impaired actions of vitamin D metabolites can result in hypophosphatemia and/ or hypocalcemia. Drugs such as diphenylhydantoin and rifampicin may cause impaired actions of vitamin D metabolites by altering vitamin D metabolism. Vitamin D-dependent rickets type 1 is caused by mutations in CYP27B1 encoding $25(\mathrm{OH}) \mathrm{D}-1 \alpha$-hydroxylase [6]. This enzyme converts $25(\mathrm{OH}) \mathrm{D}$ into $1,25(\mathrm{OH})_{2} \mathrm{D}$. Vitamin D receptor (VDR) is mutated in patients with vitamin D-dependent rickets type 2 [7]. In patients with these vitamin D-dependent rickets, both hypophosphatemia and hypocalcemia are observed. Because hypocalcemia causes secondary hyperparathyroidism resulting in reduced renal tubular phosphate reabsorp- 
tion, both impaired intestinal phosphate absorption and increased renal phosphate excretion contribute to the development of hypophosphatemia in these patients. Vitamin D deficiency is diagnosed by low serum 25(OH)D levels. Some patients with vitamin D deficiency also present secondary hyperparathyroidism. Because parathyroid hormone (PTH) stimulates conversion of $25(\mathrm{OH}) \mathrm{D}$ into $1,25(\mathrm{OH})_{2} \mathrm{D}$, patients with vitamin $\mathrm{D}$ deficiency can present various levels of $1,25(\mathrm{OH})_{2} \mathrm{D}$. Therefore, vitamin $\mathrm{D}$ deficiency cannot be diagnosed by $1,25(\mathrm{OH})_{2} \mathrm{D}$ levels.

Gastrectomy or enterectomy can cause osteomalacia [8]. It is possible that patients with osteomalacia after these surgeries are not properly diagnosed and treated. The causes for osteomalacia in these patients may be multifactorial including vitamin D deficiency and impaired mineral absorption. While liver cirrhosis or chronic liver disease was described as one of causes for osteomalacia, osteoporosis seems to be much commoner than osteomalacia in these patients [9].

There are many causes for renal phosphate wasting. About $80-90 \%$ phosphate filtered from glomeruli is absorbed in renal proximal tubules. Type $2 \mathrm{a}$ and $2 \mathrm{c}$ sodium-phosphate cotransporters mediate physiological phosphate reabsorption in proximal tubules [10]. Renal phosphate wasting can be observed in patients with Fanconi syndrome and some form of renal tubular acidosis. In addition, hereditary hypophosphatemic rickets with hypercalciuria is caused by mutations in SLC34A3 which encodes type 2c sodium-phosphate cotransporter $[11,12]$. Dent disease is characterized by low molecular weight proteinuria, hypophosphatemia and nephrolithiasis. This disease is caused by mutations in CLC5 encoding a chloride channel [13]. In addition to these intrinsic renal tubular defect, excessive actions of FGF23 cause hypophosphatemia with enhanced renal phosphate excretion. FGF23 suppresses renal tubular phosphate reabsorption by reducing the expression of type $2 \mathrm{a}$ and $2 \mathrm{c}$ sodiumphosphate cotransporters [14]. In addition, FGF23 decreases serum $1,25(\mathrm{OH})_{2} \mathrm{D}$ level by altering the expression levels of vitamin D-metabolizing enzymes [14]. Therefore, FGF23 reduces serum phosphate by suppressing renal phosphate reabsorption and inhibiting intestinal phosphate absorption through lowering $1,25(\mathrm{OH})_{2} \mathrm{D}$.

After the identification of FGF23, it became clear that patients with some kinds of hypophosphatemic rickets/osteomalacia such as X-linked hypophospha-
Table 2 FGF23-related hypophosphatemic rickets/osteomalacia

X-linked dominant hypophosphatemic rickets/osteomalacia (XLH)
Mutations in PHEX gene
Autosomal dominant hypophosphatemic rickets/osteomalacia (ADHR)
Mutations in FGF23 gene
Autosomal recessive hypophosphatemic rickets/osteomalacia 1 (ARHR1)
Mutations in DMP1 gene
Autosomal recessive hypophosphatemic rickets/osteomalacia 2 (ARHR2)
Mutations in ENPP1 gene
Hypophosphatemic disease with dental anomalies and ectopic calcification
Mutations in FAM20C gene
McCune-Albright syndrome/fibrous dysplasia
Linear sebaceous nevus syndrome

Tumor-induced rickets/osteomalacia

Hypophosphatemic rickets/osteomalacia by saccharated ferric oxide or iron polymaltose...

PHEX, phosphate-regulating gene with homologies to endopeptidases on the Xchromosome; DMP1, dentin matrix protein 1; ENPP1, ectonucleotide pyrophosphatase/phosphodiesterase 1; FAM20C, family with sequence similarity 20, member C

temic rickets $(\mathrm{XLH})$ and tumor-induced osteomalacia (TIO), a rare paraneoplastic syndrome, show high circulatory levels of FGF23 $[15,16]$. In contrast, FGF23 levels in patients suffering from other causes for rickets/osteomalacia including vitamin D deficiency were rather low [5]. Together with the biological activities of FGF23 as mentioned above, these results indicated that FGF23 is the humoral factor that causes hypophosphatemia in patients with several diseases including XLH and TIO. Since then, other hypophosphatemic diseases have also been shown to be associated with high FGF23 levels [17] (Table 2). It is believed that FGF23 production in bone is enhanced in most patients with these FGF23-related hypophosphatemic diseases except for TIO. However, the precise mechanisms of overproduction of FGF23 and the actions of gene products responsible for genetic FGF23-related hypophosphatemic diseases are largely unknown.

\section{Diagnostic Criteria (Table 3)}

Because the clinical presentations of rickets and osteomalacia are quite different, it is not practical to prepare one kind of diagnostic criteria for both of these diseases. Therefore, we propose the following diagnostic criteria (Table 3 ). Rickets is basically diagnosed by the presence of rachitic changes of bones such as cupping and fraying of metaphysis, and widening of epiphyseal plate observed by X-ray. In addition, high 
alkaline phosphatase is characteristic for diseases with impaired mineralization. We believe that these two laboratory findings are essential to make a diagnosis of rickets or suspect the presence of this disease. In addition, patients with rickets usually show hypophosphatemia or hypocalcemia, and some clinical signs. However, it is possible that patients show no clinical signs especially when the diagnosis of rickets is established in the early phase of the disease. This can happen in family members of affected patients with rickets from genetic causes. Therefore, we propose that patients with all these four findings (a to $d$ in Table 3A) are regarded to have definite rickets, and subjects who lack either hypophosphatemia or hypocalcemia, or clinical signs ( $\mathrm{c}$ or d) as possible rickets.

In contrast to rickets, there is no single laboratory test which strongly suggests the presence of osteomalacia. However, patients with osteomalacia usually show either hypophosphatemia or hypocalcemia. High alkaline phosphatase is also seen in patients with osteomalacia. While almost all alkaline phosphatase activity in blood derives from bone in children, there is significant contribution from liver and other organs in adults. We believe that either hypophosphatemia or hypocalcemia, and high bone alkaline phosphatase are essential to make a diagnosis of osteomalacia or suspect this disease. In addition, patients with osteomalacia may show some clinical symptoms, low bone mineral density (BMD), multiple uptakes by bone scintigraphy or Looser's zone. However, patients may not show these findings when osteomalacia is suspected early in the course of the disease as in the case of rickets. Therefore, we propose that patients with all these five findings (a to e in Table 3B) are regarded to have definite osteomalacia, and subjects who lack either clinical symptoms, low BMD or abnormal imaging findings (c to e) as possible osteomalacia.

Drugs that inhibit mineralization can induce rickets or osteomalacia without changing serum calcium or phosphate levels. In addition, several diseases need to be discriminated form rickets/osteomalacia (Table 4). Diseases shown in Table 4 can mimic some features of rickets or osteomalacia. On the other hand, most of these diseases cannot be diagnosed as rickets or osteomalacia using the diagnostic criteria shown in Table 3. The exceptions are multiple metastases, primary hyperparathyroidism and renal osteodystrophy. Osteoblastic bone metastases can cause either hypocalcemia or hypophosphatemia, high bone alkaline phos-
Table 3 Diagnostic criteria for rickets and osteomalacia

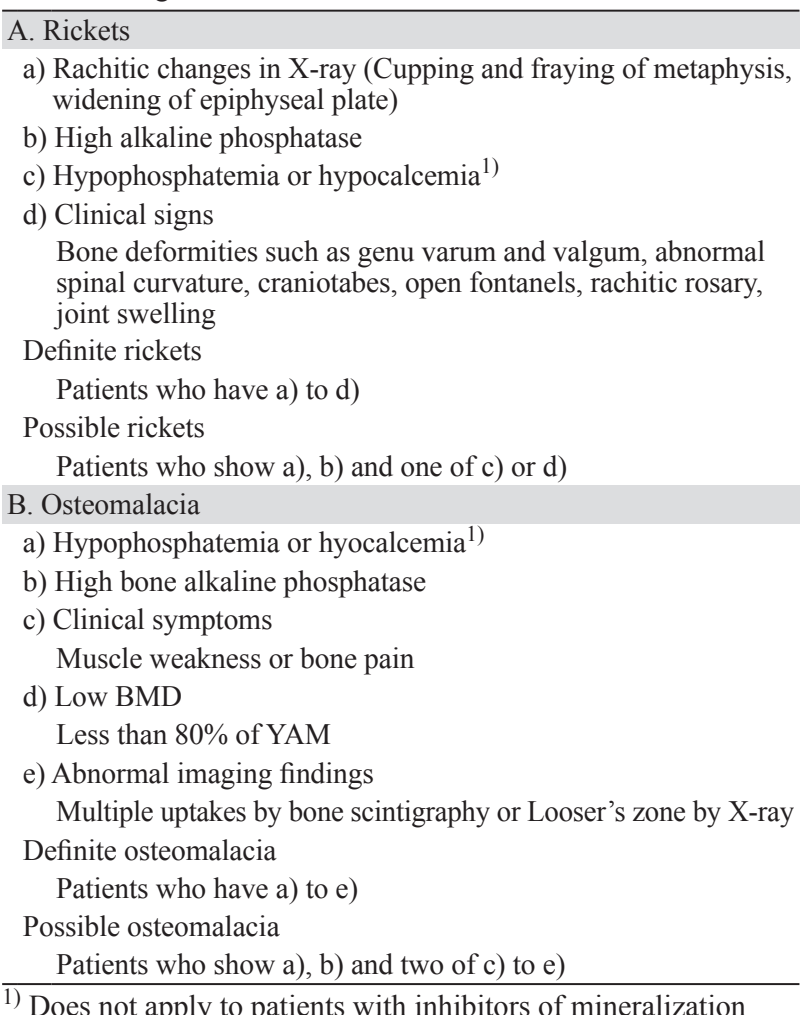

Table 4 Diseases that need to be discriminated form rickets/ osteomalacia

\begin{tabular}{l}
\hline Low BMD \\
Osteoporosis, Renal osteodystrophy, Primary \\
hyperparathyroidism \\
Bone deformity \\
Skeletal dysplasia \\
Bone pain \\
Polymyalgia rheumatica, Ankylosing spondylitis \\
Muscle weakness \\
Neuromuscular diseases \\
Multiple uptakes by bone scintigraphy \\
Multiple metastases \\
Rachitic change \\
Hypophosphatasia \\
High bone alkaline phosphatase \\
Primary hyperparathyroidism, Renal osteodystrophy, Multiple \\
metastases
\end{tabular}

phatase, bone pain and multiple uptakes by bone scintigraphy. This possibility need to be considered before finalizing the diagnosis of osteomalacia. Patients with primary hyperparathyroidism may also show hypophosphatemia, high bone alkaline phosphatase, bone pain, low BMD and multiple uptakes by bone scintigraphy. However, hypercalcemia which is rare in 
patients with osteomalacia is usually present in patients with primary hyperparathyroidism. Patients with renal osteodystrophy may show hypocalcemia, high bone alkaline phosphatase, bone pain, low BMD and multiple uptakes by bone scintigraphy. On the other hand, hyperphosphatemia rather than hypophosphatemia is usually observed in patients with renal osteodystrophy.

Hypophosphatasia is caused by mutations in TNALP which encodes tissue-nonspecific alkaline phosphatase [18]. This disease is also characterized by impaired mineralization because alkaline phosphatase converts pyrophosphate with potent inhibitory effects on mineralization into phosphate. In this sense, hypophosphatasia can be regarded as one cause for rickets. However, patients with hypophosphatasia show low alkaline phosphatase levels in contrast to those with rickets from other causes. Therefore, hypophosphatasia was treated as a disease that should be discriminated from rickets rather than a cause for rickets to avoid confusion in this proposal.

\section{Differential Diagnosis of Causes for Rickets/Osteomalacia (Table 5, Fig. 1)}

After establishing the diagnosis of rickets or osteomalacia, it is necessary to find the exact cause for these diseases. Table 5 summarizes typical biochemical changes observed in patients with various causes for rickets and osteomalacia. Vitamin D deficiency is defined by low $25(\mathrm{OH}) \mathrm{D}$ levels as mentioned before. Therefore, patients with low 25(OH)D levels are theoretically considered to have vitamin $\mathrm{D}$ deficient rickets or osteomalacia. However, vitamin D deficiency or insufficiency is quite common even in general population [19] and it is possible that low 25(OH)D levels can be observed in patients with other causes for rickets or osteomalacia. Actually, investigation of $25(\mathrm{OH}) \mathrm{D} \mathrm{lev-}$ els in patients with vitamin D deficient rickets and XLH indicated that there was overlap of serum $25(\mathrm{OH}) \mathrm{D}$ levels in these patients. In contrast, FGF23 completely discriminated patients with vitamin $\mathrm{D}$ deficient rickets and XLH [20]. From these results, we propose a flowchart for differentiating various causes for rickets and osteomalacia (Fig. 1). In patients with hypophosphatemic rickets/osteomalacia, high FGF23 levels indicate FGF23-related hypophosphatemic diseases (Table 2). Vitamin D deficient rickets/osteomalacia is diagnosed after ruling out FGF23-related hypophosphatemic diseases, phosphate depletion and other causes of renal tubular phosphate wasting. Vitamin D-dependent rickets 1 and 2 can be differentiated by $1,25(\mathrm{OH})_{2} \mathrm{D}$ levels. HHRH is also characterized by high $1,25(\mathrm{OH})_{2} \mathrm{D}$ levels. In normophosphatemic patients, drugs that inhibit mineralization and vitamin D deficiency should be considered. It should be also noted that patients with vitamin D deficiency may not show frank hypophosphatemia or hypocalcemia. It is possible that serum phosphate and calcium remain in the low normal range in these patients.

\section{Discussion}

Rickets and osteomalacia are not common diseases. However, quality of life of the affected patients can be severely compromised. For example, some untreated patients with TIO can become completely bedridden because of severe muscle weakness and bone pain. Short stature and bone deformities are big problems for patients with rickets. However, patients with rickets or osteomalacia can be completely cured or at least

Table 5 Typical biochemical findings in patient with various causes of rickets/osteomalacia

\begin{tabular}{|c|c|c|c|c|c|c|c|}
\hline & Serum $\mathrm{Ca}$ & Serum Pi & $\mathrm{TmP} / \mathrm{GFR}$ & BAP & $1,25(\mathrm{OH})_{2} \mathrm{D}$ & $25(\mathrm{OH}) \mathrm{D}$ & FGF23 \\
\hline FGF23-related hypophosphatemic disease & $\downarrow \rightarrow$ & $\downarrow$ & $\downarrow$ & $\uparrow$ & $\downarrow \rightarrow$ & $\rightarrow$ & 仓े \\
\hline Phosphate depletion & $\rightarrow$ & $\downarrow$ & $\uparrow$ & $\uparrow$ & $\rightarrow \uparrow$ & $\rightarrow$ & $\downarrow \rightarrow$ \\
\hline Fanconi syndrome & $\rightarrow$ & $\downarrow$ & $\downarrow$ & $\uparrow$ & $\downarrow \rightarrow$ & $\rightarrow$ & $\downarrow \rightarrow$ \\
\hline Vitamin D-dependent rickets type 1 & $\downarrow$ & $\downarrow$ & $\downarrow$ & $\uparrow$ & $\downarrow$ & $\rightarrow$ & $\downarrow \rightarrow$ \\
\hline Vitamin D-dependent rickets type 2 & $\downarrow$ & $\downarrow$ & $\downarrow$ & $\uparrow$ & $\uparrow$ & $\rightarrow$ & $\downarrow \rightarrow$ \\
\hline HHRH & $\rightarrow$ & $\downarrow$ & $\downarrow$ & $\uparrow$ & $\uparrow$ & $\rightarrow$ & $\downarrow \rightarrow$ \\
\hline Vitamin D deficiency & $\downarrow \rightarrow$ & $\downarrow \rightarrow$ & $\downarrow \rightarrow$ & $\uparrow$ & $\rightarrow \uparrow \downarrow$ & ת & $\downarrow \rightarrow$ \\
\hline Drugs that inhibit mineralization & $\rightarrow$ & $\rightarrow$ & $\rightarrow$ & $\uparrow$ & $\rightarrow$ & $\rightarrow$ & $\rightarrow$ \\
\hline
\end{tabular}

沧: Specific laboratory tests for each category.

$\mathrm{Pi}$, phosphate; TmP/GFR, tubular maximum transport of phosphate per glomerular filtration rate; BAP, bone alkaline phosphatase; $1,25(\mathrm{OH})_{2} \mathrm{D}, 1,25$-dihydroxyvitamin $\mathrm{D} ; 25(\mathrm{OH}) \mathrm{D}$, 25-hydroxyvitamin $\mathrm{D}$; HHRH, hereditary hypophosphatemic rickets with hypercalciuria 


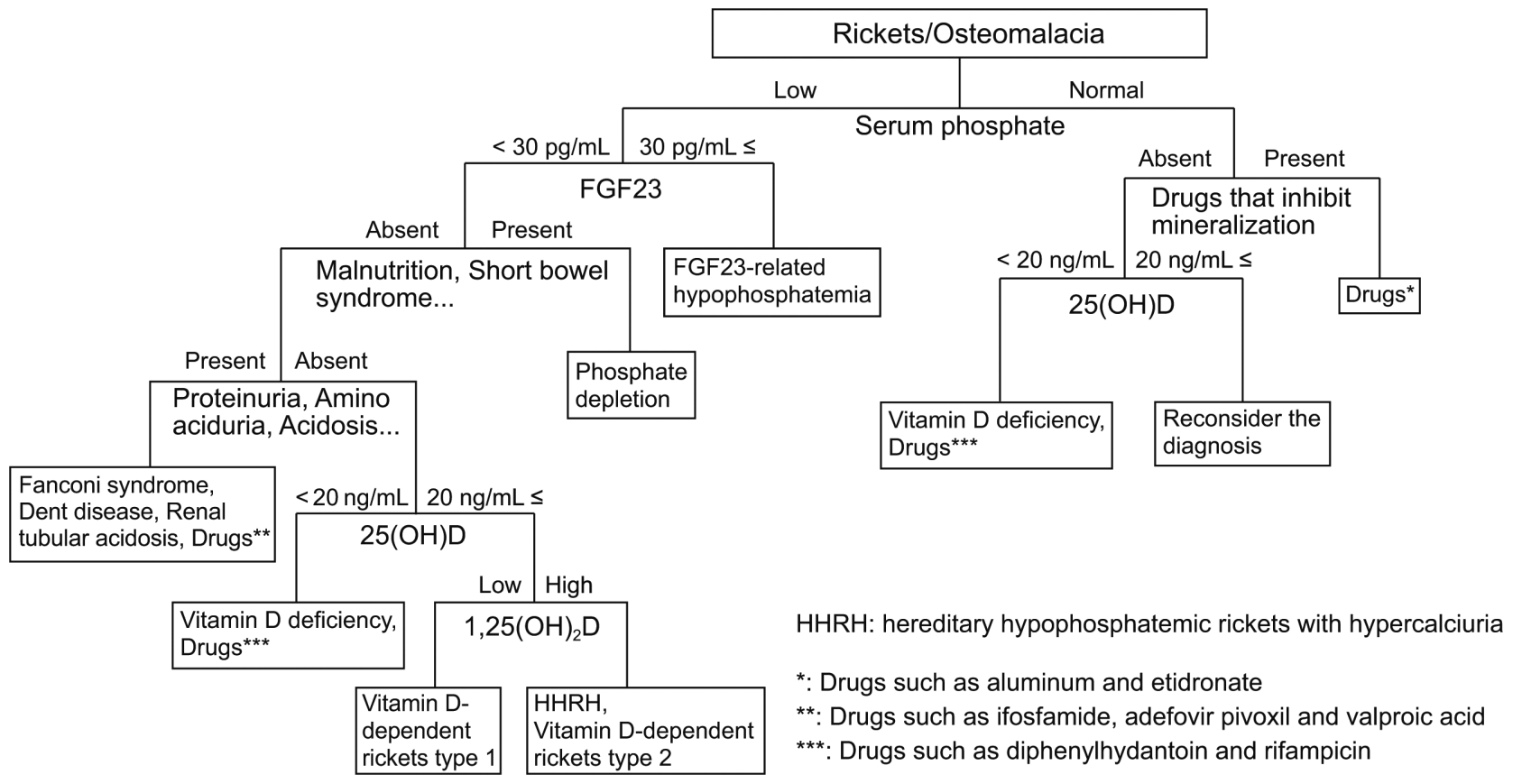

Fig. 1 A flowchart for the differential diagnosis of causes for rickets/osteomalacia

The causes for rickets and osteomalacia can be identified by several clinical findings and laboratory tests.

respond to treatment when properly diagnosed and treated according to the specific causes. Therefore, we here listed causes for rickets and osteomalacia, and proposed the diagnostic criteria and a flowchart for the differential diagnosis of various causes for these diseases.

There are several limitations in the proposed diagnostic criteria. These criteria are not created by retrospective review of clinical presentations of a large number of patients, but proposed by several researchers and clinicians based on their experiences. Therefore, the validity of these criteria and the flowchart needs to be examined in the future studies. However, without any diagnostic criteria, it would be difficult for general medical professionals to correctly diagnose not-so-common illnesses. We hope that this proposal will become a momentum for propagation of proper knowledge about rickets and osteomalacia, and for accumulation of more clinical data for future revision of the criteria. In addition, there was a discussion about hypophosphatasia among us. This disease can be considered to be one cause of rickets. However, if hypophosphatasia is included in the causes for rickets, high alkaline phosphatase cannot be used as one of criteria for the diagnosis of rickets, and the flowchart for the differential diagnosis of various causes needs to be more complex. Because hypophosphatasia is rarer than other causes for rickets such as vitamin D deficiency and XLH, and the easily usable diagnostic criteria for rickets and osteomalacia were planned, hypophosphatasia was not included as a cause for rickets in this proposal. Finally, measurements of FGF23 and 25(OH)D are not covered by medical insurance in Japan now and are not routine laboratory tests. In contrast, these measurements are available in several commercial and research laboratories. We hope that this proposal will contribute to some extent to the future coverage of these measurements by medical insurance in Japan.

In summary, we have created a diagnostic criteria and a flowchart for the differential diagnosis of various causes for rickets/osteomalacia. We hope that these criteria and flowchart are clinically useful for the proper diagnosis and management of patients with these diseases.

\section{Acknowledgements}

This study was supported in part by a grant from Ministry of Health, Labour and Welfare of Japan.

\section{Disclosure}

The authors declare no conflicts of interest. 


\section{References}

1. Reginato AJ, Coquia JA (2003) Musculoskeletal manifestations of osteomalacia and rickets. Best Pract Res Clin Rheumatol 17: 1063-1080.

2. Whyte MP, Thakker RV (2009) Rickets and osteomalacia. Medicine 37: 483-488.

3. Prentice A (2013) Nutritional rickets around the world. J Steroid Biochem Mol Biol 136: 201-206.

4. Fukumoto S, Martin TJ (2009) Bone as an endocrine organ. Trends Endocrinol Metab 20: 230-236.

5. Endo I, Fukumoto S, Ozono K, Namba N, Tanaka H, et al. (2008) Clinical usefulness of measurement of fibroblast growth factor 23 (FGF23) in hypophosphatemic patients: proposal of diagnostic criteria using FGF23 measurement. Bone 42: 1235-1239.

6. Kitanaka S, Takeyama K, Murayama A, Sato T, Okumura K, et al. (1998) Inactivating mutations in the 25-hydroxyvitamin D3 1alpha-hydroxylase gene in patients with pseudovitamin D-deficiency rickets. $N$ Engl J Med 338: 653-661.

7. Hughes MR, Malloy PJ, Kieback DG, Kesterson RA, Pike JW, et al. (1988) Point mutations in the human vitamin D receptor gene associated with hypocalcemic rickets. Science 242: 1702-1705.

8. Tovey FI, Hall ML, Ell PJ, Hobsley M (1992) A review of postgastrectomy bone disease. $J$ Gastroenterol Hepatol 7: 639-645.

9. Collier J (2007) Bone disorders in chronic liver disease. Hepatology 46: 1271-1278.

10. Blaine J, Chonchol M, Levi M (2015) Renal control of calcium, phosphate, and magnesium homeostasis. Clin J Am Soc Nephrol 10: 1257-1272.

11. Bergwitz C, Roslin NM, Tieder M, Loredo-Osti JC, Bastepe M, et al. (2006) SLC34A3 mutations in patients with hereditary hypophosphatemic rickets with hypercalciuria predict a key role for the sodium-phosphate cotransporter NaPi-IIc in maintaining phosphate homeostasis. Am J Hum Genet 78: 179-192.

12. Lorenz-Depiereux B, Benet-Pages A, Eckstein G,
Tenenbaum-Rakover Y, Wagenstaller J, et al. (2006) Hereditary hypophosphatemic rickets with hypercalciuria is caused by mutations in the sodium-phosphate cotransporter gene SLC34A3. Am J Hum Genet 78: 193-201.

13. Lloyd SE, Pearce SH, Fisher SE, Steinmeyer K, Schwappach B, et al. (1996) A common molecular basis for three inherited kidney stone diseases. Nature 379: 445-449.

14. Shimada T, Hasegawa H, Yamazaki Y, Muto T, Hino R, et al. (2004) FGF-23 is a potent regulator of vitamin D metabolism and phosphate homeostasis. J Bone Miner Res 19: 429-435.

15. Jonsson KB, Zahradnik R, Larsson $\mathrm{T}$, White KE, Sugimoto T, et al. (2003) Fibroblast growth factor 23 in oncogenic osteomalacia and X-linked hypophosphatemia. N Engl J Med 348: 1656-1663.

16. Yamazaki Y, Okazaki R, Shibata M, Hasegawa Y, Satoh $\mathrm{K}$, et al. (2002) Increased circulatory level of biologically active full-length FGF-23 in patients with hypophosphatemic rickets/osteomalacia. J Clin Endocrinol Metab 87: 4957-4960.

17. Fukumoto S (2014) Anti-fibroblast growth factor 23 antibody therapy. Curr Opin Nephrol Hypertens 23: 346-351.

18. Weiss MJ, Cole DE, Ray K, Whyte MP, Lafferty MA, et al. (1988) A missense mutation in the human liver/ bone/kidney alkaline phosphatase gene causing a lethal form of hypophosphatasia. Proc Natl Acad Sci U S A 85: 7666-7669.

19. Bendik I, Friedel A, Roos FF, Weber P, Eggersdorfer M (2014) Vitamin D: a critical and essential micronutrient for human health. Front Physiol 5: 248.

20. Kubota T, Kitaoka T, Miura K, Fujiwara M, Ohata Y, et al. (2014) Serum fibroblast growth factor 23 is a useful marker to distinguish vitamin D-deficient rickets from hypophosphatemic rickets. Horm Res Paediatr 81: 251257. 